\title{
Las prácticas maternas frente a la enfermedad diarreica infantil y la terapia de rehidratación oral
}

\author{
Selene Alvarez-Larrauri, D r. en So (1) $^{(1)}$
}

\begin{abstract}
Alvarez-Larrauri S. Las prácticas maternas frente a la enfermedad diarreica infantil y la terapia de rehidratación oral. Salud Publica Mex 1998;40:256-264.
\end{abstract}

\begin{abstract}
Resumen
Objetivo. D eterminar las prácticas terapéuticas utilizadas por madres de Coatepec, Veracruz, cuando se presentan episodios diarreicos en sus hijos menores de cinco años, su grado de generalización, así como su relación con los entramados de significados sociales que las sustentan. Material y métodos. De un total de 15740 familias, se seleccionó aleatoriamente una muestra de 300. Asimismo, se hizo un levantamiento de información cuantitativa y cualitativa a partir de entrevistas abiertas estucturadas. Se llevó a cabo un análisis estadístico (no paramétrico) de las prácticas terapéuticas y se estudió su relación con variables socioeconómicas. Resultados. Fueron frecuentes las dos prácticas consideradas como las más importantes en la obstaculización de la terapia de rehidratación oral: bajo uso del sobre de hidratación oral (SHO) y medicación contraindicada. El análisis no paramétrico indicó una relación de significancia entre las prácticas contraindicadas y el hecho de no tener claro el concepto de deshidratación. Por otro lado, se encontró que existe una relación de significancia entre tener el concepto correcto de deshidratación y: a) usar el $\mathrm{SHO}$; b) no usar medicamentos contraindicados, y c) utilizar menos la medicina tradicional. Las variables socioeconómicas no tuvieron relación de significancia con las prácticas ni con el hecho de saber lo que significa la deshidratación. Se describe, con base en la interpretación cualitativa, cómo se construyen los significados que sustentan estas prácticas y cómo se reproducen por medio de las redes sociales. Conclusiones. Se recomienda investigar también la participación de los proveedores de salud en la reproducción de las prácticas terapéuticas, así como la relación que ello guarda con los patrones de reproducción de los significados en las redes sociales de ayuda.
\end{abstract}

Palabras clave: diarrea infantil; conducta materna; terapia de rehidratación oral; práctica profesional; México re

(1) Investigadora del Instituto $\mathrm{N}$ acional de Antropología e Historia, México.

Fecha de recibido: 10 de septiembre de 1997 - Fecha de aprobado: 13 de marzo de 1998

Solicitud de sobretiros: Dra. Selene Alvarez. Instituto N acional de Antropología e Historia. A partado Postal 639, 91000 Xalapa, Veracruz, México.

\author{
Alvarez-Larrauri S. \\ Maternal practice in case \\ of infantile diarrhea \\ and oral rehydration therapy. \\ Salud Publica Mex 1998;40:256-264.
}

\begin{abstract}
Objective. To determine therapeutic practices used by mothers from Coatepec,Veracruz, in case of diarrheic episodes in their children under 5 years of age, the degree of generalization, and the relationship with the social frame that sustains them. Material and methods. A sample of 300 families was rando mly selected from a total of 15740 . Q uantitative and qualitative information was gathered through structured open surveys. N on-parametric statistical analysis of the therapeutic practices was performed and their relationship with socioeconomic variables was analyzed. Results. The two practices considered to obstruct the oral rehydration therapy most, were common: infrequent use of oral rehydration serum (ORS) and contraindicated medication. N on-parametric analysis indicated a significant relationship between contraindicated practices and a mistaken concept of dehydration. $0 \mathrm{n}$ the other hand, the relationship between a correct concept of dehydration and a) using $O R S, b$ ) not using contraindicated medication and c) limiting the use of traditional medicine was also significant. So cioeconomic variables had no significant relationship with any particular practice nor with the concept of dehydration. $Q$ ualitative interpretation describes how the social meanings that sustain these practices are constructed and reproduced through social nets. Conclusions The participation of health suppliers in the reproduction of therapeutic practices should be further investigated, as well as their relationship with the reproduction patterns of social meaning through the nets of social aid.
\end{abstract}

Key words: diarrhea, infantile; maternal behavior; oral rehydration therapy; professional practice; Mexico 
$\mathrm{E}$ n México la mortalidad por diarrea sigue ocupando el primer lugar como enfermedad infecciosa, tanto en el grupo prescolar como en el infantil (menores de un año); ${ }_{i}^{1}$ dentro de este último grupo en particular tiene el tercer lugar, después de los padecimientos perinatales y congénitos. Durante 1993 se registraron 6748 defunciones en el país por enfermedades diarreicas, de tal modo que la tasa de mortalidad infantil por esta causa fue de 60.4 por cada 100000 menores de cinco años, mientras que en el estado de Veracruz fue de 54.4. ${ }^{2}$

Más de $70 \%$ de estas muertes puede evitarse si se utiliza la terapia de rehidratación oral, que consiste en incrementar el consumo de líquidos, dar suero de hidratación oral (SHO), continuar la alimentación y no usar antidiarreicos. ${ }^{3}$ La mayoría de los episodios de diarrea manejados de esta manera evolucionan favorablemente y no necesitan atención médica. ${ }^{*}$

Diversas investigaciones indican que hay dos situaciones que impiden que esa terapia evite la deshidratación y los desenlaces fatales: la primera tiene que ver con el escaso uso del SHO, y la segunda, con el consumo de medicinas contraindicadas. Así, por ejemplo, la Encuesta sobre el Manejo Efectivo de los Casos de Diarrea de la Secretaría de Salud en Veracruz reporta que en este estado, en 1993, el uso de SHO en los episodios diarreicos fue de $56.7 \%$ y que de ese porcentaje sólo $60.9 \%$ lo usó adecuadamente. ${ }^{4}$ La misma encuesta, en el ámbito nacional, notifica que en $17.6 \%$ de los casos de diarrea se suministraron antidiarreicos y en $56.2 \%$ de los casos se utilizaron antibióticos, lo cual representa una cifra muy elevada considerando que la mayoría de las diarreas agudas se deben a infecciones virales autolimitadas cuya evolución no se modifica con el empleo de antibióticos.

En lo que se refiere a los médicos institucionales y particulares se ha visto que no siempre incluyen en el tratamiento de la diarrea el SHO, y la mayoría prefiere utilizar antibióticos, antieméticos, antidiarreicos y antiparasitarios. ${ }^{5}$ Esta situación se repite en los casos de los niños que han muerto por deshidratación. En Veracruz, las autopsias verbales llevadas a cabo por la Secretaría de Salud indican que la mayoría de los niños recibieron atención médica durante el episodio que derivó en desenlace fatal, sin que se les suministrara SHO; además, se les prescribieron medicamentos contraindicados. Apuntan también que un número importante de niños no regresaron a la consulta ante los

\footnotetext{
* Organización Mundial de la Salud. Programme for control of diarrhoeal diseases. Interim programme report. Ginebra: OMS, 1992: 42. Documento no publicado.
}

signos de deshidratación y estado de gravedad, o bien, fueron llevados demasiado tarde como para poder salvarlos. Destacan, asimismo, que la mayoría de las muertes ocurrió en el hogar y que las situaciones fueron también manejadas por la familia con autoprescripción incorrecta y sin usar el SHO. * Se ha documentado que el uso de medicamentos contraindicados ${ }^{\ddagger}$ es, en promedio, dos a cuatro veces más frecuente que el uso del SHO, y no es raro que los médicos o farmacéuticos receten, o bien, que las madres autorreceten cuatro o más medicinas en un solo episodio. ${ }^{6}$

Las razones por las cuales persisten las prácticas de medicación incorrecta y de no recurrir al SHO en la población han sido poco estudiadas. Algunas investigaciones han encontrado una relación significativa entre condiciones sociales y variables culturales, por un lado, y el rechazo del SHO, por el otro. Por ejemplo, se afirma que: "las familias clasificadas como tradicionales rechazaron con mayor frecuencia este tipo de tratamiento" ${ }^{7}$ o que "las madres que tienden a subestimar la deshidratación son de nivel económico más bajo y tienen menor acceso a los servicios médicos y niños más desnutridos" ${ }^{8}$ Por otro lado, se ha descrito el proceso de toma de decisiones durante las enfermedades diarreicas para poder entender el manejo en el hogar por parte de la familia. A partir del reconocimiento de que el niño sufre de diarrea, las prácticas se dividen en dos fases: en la primera se realiza una serie de prácticas de autoatención esperando que se cure la enfermedad; en la segunda fase, el episodio se hace "público", es decir, la familia empieza a preocuparse porque no cede la enfermedad y determina que se requiere otro tipo de atención. ${ }^{9}$ El paso a la segunda fase se da en los casos en que no se ha detenido la diarrea (cabe recordar que la diarrea es autolimitada y dura de tres a cinco días) y empieza a complicarse con deshidratación. En esta fase se realiza una serie de prácticas curativas, delimitadas por la combinación de recursos que ofrece el entorno social con los que tiene la

\footnotetext{
* Servicios Coordinados de Salud Pública en el Estado de Veracruz. Resultados de autopsias verbales, enero-diciembre 1993. Informe del Secretario Técnico. Xalapa: Secretaría de Salud en el Estado de Veracruz, 1993. Documento no publicado.

₹ La administración de antidiarreicos y antibióticos tiene muchos inconvenientes, sobre todo desvía la atención del tratamiento correcto de la enfermedad, es decir, de la prevención de la deshidratación. Otro problema es que el uso de los llamados medicamentos antidiarreicos frecuentemente se asocia con efectos colaterales indeseables que pueden ocasionar inclusive la muerte. Además, el abuso de los antibióticos es causa de diarreas prolongadas. La medicación incorrecta ha sido documentada también como una de las prácticas más difíciles de cambiar en los programas de educación para la salud.
} 
familia: medicación contraindicada recomendada por las redes de ayuda o farmacéuticos, así como acudir al médico privado, al tradicional o al institucional. Algunas madres que han tenido hijos severamente deshidratados han descrito cómo van combinando indiscriminadamente las distintas prácticas conforme se agrava la situación. El patrón de estas combinaciones es el de utilizar todo tipo de recurso que se tiene a la mano y cada vez con mayor desesperación, pues lo que se hace no está solucionando el problema, no se está "curando" la diarrea. Se da entonces la paradoja de realizar toda una serie de prácticas para curar la diarrea -que no es lo peligroso-, en vez de prevenir la deshidratación, que es la que causa la muerte. ${ }^{10}$

Se ha visto que esta costumbre de recurrir a las prácticas "curativas de la diarrea" está relacionada con el concepto que tienen las madres acerca de la diarrea y la deshidratación: confunden ambos conceptos pensando que la deshidratación es sólo el conjunto de síntomas de la diarrea y que lo que hay que hacer es "pararla". Desconocen que la deshidratación es un proceso derivado de la diarrea, una complicación de la misma, que requiere de atención específica. En una investigación cualitativa llevada a cabo en Veracruz con madres de hijos con deshidratación severa, se vio que sólo $2 \%$ del total de madres entrevistadas describieron la deshidratación como "pérdida de líquidos y sales minerales". ${ }^{5}$ Según esa investigación cualitativa, la confusión entre diarrea y deshidratación está vinculada a la práctica de no utilizar el $\mathrm{SHO}$, pero también a la de suministrar medicamentos contraindicados. Incluso, aumentar el consumo de líquidos -mediante la administración de tés, refrescos y atoles- no se asocia generalmente con la reposición de líquidos necesaria para prevenir la deshidratación, sino con la curación de la diarrea.

A partir de las investigaciones descriptivas se optó por determinar qué tan generalizada es la confusión entre diarrea y deshidratación y cuál es su relación con las prácticas de medicación contraindicada, medicación con medicina tradicional y uso del SHO. Asimismo, era importante conocer el significado que dan a sus prácticas las personas encargadas de atender a los niños con diarrea, para poder entender por qué muchas de ellas recurren a prácticas proscritas, por un lado, y no llevan a cabo las que garantizarían el manejo correcto de la enfermedad, por el otro. Se consideró que saber qué tan generalizada está esa situación y entender de dónde se deriva y cómo se reproduce por medio de las redes sociales sería de gran utilidad a los esfuerzos educativos del sector salud; se podrían incorporar los significados sociales que permiten a la familia relacionar el uso del SHO con la prevención de la deshidratación -desvinculándola del proceso curativo de la diarrea- y entender lo innecesario de los medicamentos en las diarreas agudas.

Así, los objetivos de la investigación fueron: determinar las prácticas terapéuticas utilizadas durante los distintos episodios de diarrea reportados por las madres, su grado de generalización y cómo se relacionan entre ellas; determinar si las madres saben lo que es la deshidratación y si esa situación obstaculiza el uso del SHO y alienta el de la medicación incorrecta, y por último, comprender los significados que las personas atribuyen a las prácticas terapéuticas y cómo se reproducen éstas, junto con los significados, en el tejido social.

\section{Material y métodos}

Se diseñó una encuesta aleatoria, para hacer el levantamiento de datos a partir de entrevistas cara a cara. Para disminuir la dificultad que plantea la entrevista abierta en la identificación de rasgos -debido a su falta de estructura-, se optó por una entrevista abierta estructurada con una guía de preguntas que permitiera el registro tanto de la información sujeta al análisis cuantitativo, como de aquella que sería objeto de interpretación (la información generada en el proceso de reflexión). Se excluyó el uso de un cuestionario, pues parte de la información que había que recolectar debía ser una construcción mental previa a la acción, que la misma entrevistada podía incluso no haber elaborado, mientras que a partir de una pregunta cerrada sólo había posibilidad de obtener una respuesta manifiesta. Por ejemplo, al preguntar qué acciones se realizaron en determinado episodio, la persona tiene la referencia de la experiencia previa de acciones específicas y puede reportarlas de manera clara mientras que, al indagar sobre un significado (construcción mental previa a la acción), no se tiene una referencia concreta sino que es necesario recurrir a la reflexión (proceso hermenéutico) y a la interpretación de las acciones junto con la entrevistada.

El universo de trabajo estuvo constituido por las madres que residen en Coatepec, Veracruz (15 740 familias). Del número total se tomó una muestra aleatoria de 300 madres de niños menores de cinco años. Este estudio se llevó a cabo del 12 de julio al 1 de agosto de 1994.

La muestra se seleccionó de la siguiente manera: en un mapa proporcionado por la oficina de obras públicas del municipio, se numeraron las cuadras de la ciudad para que todas tuvieran la posibilidad de salir 
en la selección aleatoria. Se dividió el número total de viviendas en la ciudad (Censo Nacional de 1990) entre el número de cuadras registradas en el mapa para obtener el número aproximado de casas por cuadra. Se dividió el número de entrevistas que era posible hacer en el lapso dedicado al trabajo de campo, entre el número de viviendas por cuadra, y resultó el número de cuadras que era necesario seleccionar aleatoriamente para completar ese número de entrevistas. Se verificó que ese número fuera representativo para poder generalizar. Para garantizar que se encontraría el número necesario de madres con niños menores de cinco años se aumentó un 30\% de cuadras, con el fin de poder descontar las viviendas donde no hubiera niños menores de cinco años, o bien, hubiera niños que no se hubiesen enfermado en el lapso por incluir (11 meses).

De este modo, se realizaron 300 entrevistas de tipo abierto con una guía de preguntas generadoras; sin embargo, se desecharon 31 entrevistas por no tener la posibilidad de precisar las variables a partir de las mismas. Participaron dos pedagogas, un antropólogo y una socióloga en la recopilación de la información.

Se registró la información concerniente a las prácticas -y los significados que les atribuían las entrevistadas- realizadas en un episodio recordado con claridad y que hubiera sucedido en los últimos 11 meses, mediante diario de campo y grabación de las entrevistas. Se hizo un análisis estadístico (no paramétrico) de las prácticas y de si se tenía el concepto correcto de deshidratación, así como de la relación entre éstos. Se analizó también la relación de los anteriores aspectos con las variables socioeconómicas. Los datos fueron codificados, capturados y analizados en el programa EPI INFO. Se sacaron frecuencias y pruebas de significancia entre las distintas variables.

Los significados atribuidos por las entrevistadas a las distintas acciones se trataron mediante procedimientos basados en la llamada teoría fundamentada. Se les codificó (codificación abierta y axial) y se hizo la descripción del patrón de reproducción y generalización dentro del tejido social. Los principales códigos que surgieron fueron: representaciones sociales en relación con acciones; intencionalidades en relación con acciones; relación de la acción decidida con los recursos existentes; relación de las acciones con la participación de familiares; relación entre acciones y proveedores médicos; reproducción de representaciones y prácticas, complejos tradicionales (frío-caliente, empacho, etc.), ansiedad, responsabilidad y sensación de ser competente para la atención.

\section{Marco teórico}

Las prácticas de los sujetos sociales son subjetivas y, al mismo tiempo, se repiten dentro del tejido social y establecen patrones regulares, esto es, pueden "objetivarse". Las prácticas son acciones que realizan las personas porque están referidas a un sentido, a un significado. Estos significados tienen la peculiaridad de que, a la vez que son individuales (ya que están basados en vivencias anteriores), están circunscritos histórica y socialmente, o sea, en determinadas condiciones sociales y culturales. ${ }^{11}$ La elaboración de significados es una relación abierta entre el individuo que construye su intersubjetividad, inserto en una dimensión social que lo cobija; realiza prácticas que tienen significado a la vez que esta acción reproduce los significados. Las prácticas se basan en la autocomprensión de los individuos en términos de valores, símbolos, saberes, significados, etcétera, intersubjetivamente compartidos. Son parte de una intersubjetividad los sujetos que participan en los sistemas de representación social que, a su vez, les permiten obtener representaciones de la realidad; estas últimas son "el conjunto de procesos articulados de percibir, categorizar y significar los modos globales de interpretar, de asignar sentidos en la vida social" ${ }^{12}$ Estos sentidos son interiorizados a partir de la relación particular del individuo con la realidad, mediante el lenguaje, la experiencia, la pertenencia a un grupo, las prácticas que realiza y su interacción con los "otros".

En este trabajo las prácticas se conciben como distintas y regulares dentro de un determinado tejido social, y debido a esa característica pueden ser analizadas e interpretadas a la vez.

\section{Variables}

Sujeto/madre. Para los fines de este estudio, se hace referencia a aquellas mujeres residentes en Coatepec que tienen hijos menores de cinco años y que realizaron prácticas en torno a un episodio de diarrea específico.

Prácticas de atención a la diarrea aguda infantil. Acciones privilegiadas por parte de la madre y realizadas al momento de reconocer el episodio de diarrea de un menor de cinco años, que hayan tenido lugar en los últimos 11 meses, independientemente del desarrollo y el desenlace del episodio:

1. Práctica de uso de medicamentos. Recurrencia a la medicación incorrecta contraindicada en los ca- 
sos de diarrea aguda infantil y/o uso de medicamentos prescritos por un médico o farmacéutico en algún episodio de diarrea.

2. Práctica de uso de medicina tradicional. Recurrencia al uso de "tés", de cura de "empacho", de "caída de mollera" y de "susto".

3. Práctica de uso del SHO. Recurrencia a la administración de suero de hidratación oral al niño, en cualquier momento del episodio.

Representaciones y significados. Significados que dan las personas a sus prácticas al reflexionar sobre las mismas.

Concepto de deshidratación. Si la madre sabía que la deshidratación es la pérdida de los líquidos que el organismo necesita para su funcionamiento vital o si le atribuye cualquier otro significado.

Vivienda. Variable de control que se refiere al tipo de vivienda según los materiales utilizados: buena si tenía techo, piso y paredes de cemento y tabique; regular si tenía, por lo menos, dos de estos elementos; mala si carecía de dos de estos materiales.

Edad. Variable de control que se refiere a los años cumplidos por la madre al momento de la entrevista.

\section{Resultados}

\section{Prácticas terapéuticas}

Resultaron frecuentes las dos prácticas que se han determinado como las más importantes en la obstaculización de la terapia de rehidratación oral: bajo uso del SHO y medicación contraindicada.

Del total de madres entrevistadas, 125 (47\%) proporcionaron $\mathrm{SHO}$ y $144(53 \%)$ no lo dieron. La práctica de usar medicamentos fue la siguiente: $57 \%$ del total de las madres proporcionaron medicamentos contraindicados a los niños enfermos. Solamente cinco madres $(.03 \%)$ dijeron haber acudido a consulta en alguna institución o con un médico privado como primera acción antes de proporcionar el medicamento, lo cual indica una extendida automedicación en casa durante la primera fase. Algunas madres acudieron al médico (principalmente a particulares) o al farmacéutico durante la segunda fase del episodio para comprar el medicamento, pero no para consulta. Al parecer, es una práctica común ir al médico o farmacéutico, sin llevar al niño, ya que si lo llevan se les cobra la consulta, mientras que si sólo le explican lo que tiene y obtienen la receta médica, sólo esta última hay que pagar. El hecho de que se hayan recetado medicamentos contraindicados en las diarreas agudas y, en varios casos, sin ver al niño, indica que el fenómeno de la automedicación en casa es una práctica que está relacionada con la práctica profesional de los proveedores de servicio, quienes están proporcionando el medicamento sin que medie la consulta o la están vendiendo sin prescripción médica. Los medicamentos contraindicados más utilizados fueron, en orden de importancia, acromicina, terramicina y penicilina, Kaomicyn, Kaopectate y Peptobismol.

La práctica de uso de medicina tradicional apareció básicamente como paliativo o como recurso barato cuando el episodio se fue complicando y no como obstáculo al uso del SHO. Se encontró que los tés fueron el recurso más utilizado en relación con la medicina tradicional, aunque fue una práctica no tan recurrente como se había esperado: del total de madres solamente $46(17 \%)$ proporcionaron tés. La medicina tradicional se utilizó como un complemento de medicamentos contraindicados o del SHO, especialmente cuando se determinaba que no se daban los resultados esperados, es decir, que se quitara la diarrea. Asimismo se utilizó en casos en los que la familia no tenía dinero para ir al médico o a la farmacia para comprar la medicina y autoadministrarla. Finalmente, la cura de empacho en diarrea aguda se limitó a un caso.

Por lo que se refiere a la relación entre las distintas prácticas, resultó que 30\% de las 125 madres que sí dieron el SHO utilizaron medicinas contraindicadas también ( $14 \%$ del total de madres entrevistadas). El porcentaje de madres que utilizó medicamentos, dentro del grupo de 144 que no usó SHO, fue de 70 (43\% del total). Fue mayor el porcentaje de madres que usó medicamentos en el grupo que no utilizó el SHO que en el que sí lo administró (figura 1).

El análisis estadístico comprobó que existe una relación significativa entre el uso del SHO y el de medicamentos: a mayor administración de medicamentos, menor uso del SHO ( $p=0.00000000)$.

Además de administrar el SHO, 7 de las 125 madres de este grupo (6\% del total) también utilizaron tés. Dentro del grupo de las que no utilizaron el SHO, 30 madres (11\% del total) recurrieron a tés (figura 1). Aquí también apareció una relación entre uso de suero y uso de medicina tradicional. Sin embargo, no se trata de una relación de significancia estadística. Por otro lado, sí apareció como significativa $(p=0.00000000)$ la relación entre el uso de medicamentos y el uso de medicina tradicional.

\section{Diarrea versus deshidratación}

Por lo que se refiere a si las madres confunden diarrea con deshidratación, sólo 62 (23\%) sabían con precisión qué significa este último término: lo describieron como 


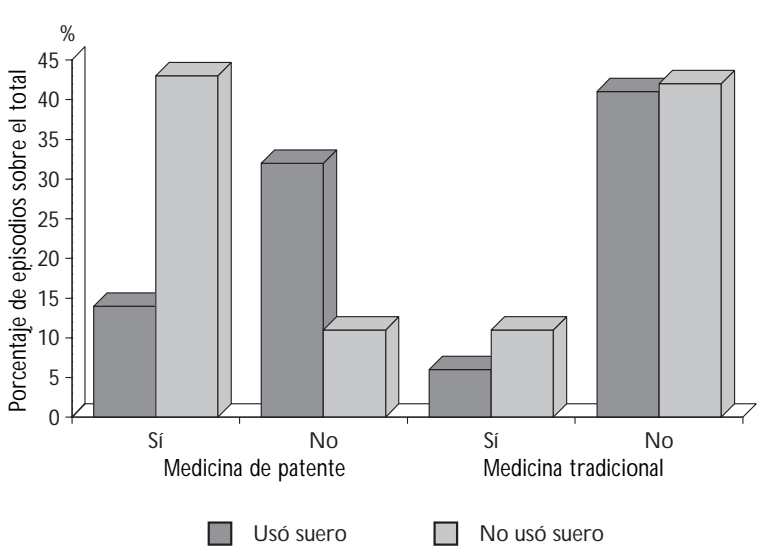

Figura 1. Uso del suero en relación con el uso de medicina de patente y medicina tradicional, CoatePeC, Veracruz, 1994

"pérdida de líquidos". El resto del grupo la describió como: "debilidad", "cuando dejan de comer", "cuando dejan de beber", "se adelgazan", "están pálidos", "se les sume la mollera", "tienen los ojos hundidos", "tienen sed", "lloran sin lágrimas" y "tienen la boca seca".

De las 125 madres que administraron el SHO, 53 (42\%) tenían el concepto correcto de deshidratación; de éstas, 44 (83\% del total) dijeron que lo habían dado para evitarla. Las otras nueve madres $(17 \%)$ administraron el SHO siguiendo la instrucción de otra persona, sin tener claro el concepto de deshidratación (figura 2).

El hecho de tener el concepto correcto de deshidratación, por un lado, y utilizar el SHO, por el otro, presentó en el análisis de resultados una significancia estadística de $p=0.00000000$.

Tener claro el concepto de deshidratación tuvo también una significancia estadística $(p=0.00000138)$ con una menor utilización de medicamentos contraindicados. Asimismo, el cruce entre tener el concepto correcto de deshidratación y una menor utilización de medicina tradicional fue también significativo: $p=$ 0.00148840 (figura 3).

El cruce de las variables con las de control no determinó ninguna relación de significancia entre la edad de la madre y el tipo de vivienda, ${ }^{*}$ por un lado, y las

\footnotetext{
* Las madres tuvieron de 15 a 25 años de edad en $22 \%$ de los casos; de 25 a 35 años en $54 \%$, y $24 \%$ fueron mayores de 35 años. De las viviendas, $66 \%$ fueron buenas; $22 \%$, regulares, y $12 \%$, malas.
}

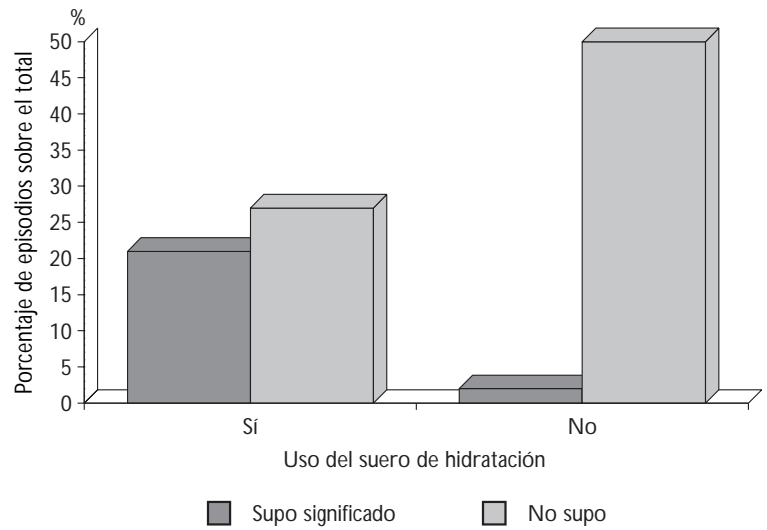

Figura 2. Relación entre tener el Concepto correcTO DE DESHIDRATACIÓN Y USAR EL SUERO DE HIDRATACIÓN oral, Coatepec, Veracruz, 1994

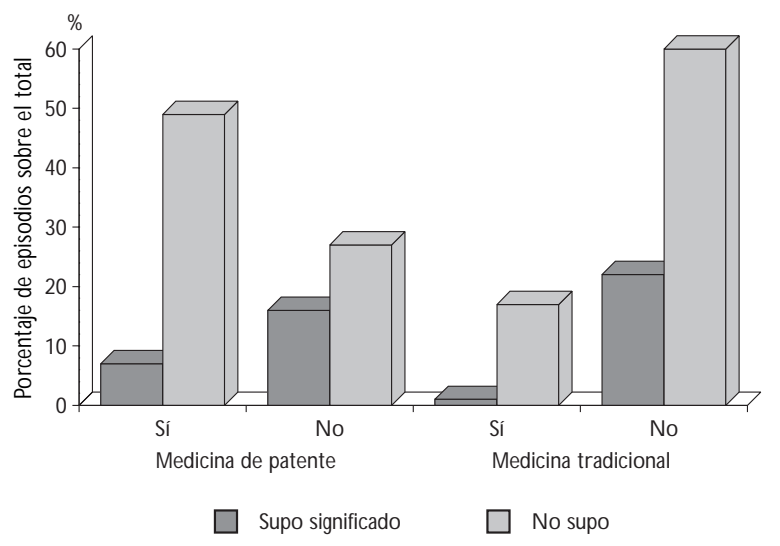

Figura 3. Relación entre tener el CONCEPTO CORRECTO DE DESHIDRATACIÓN Y EL USO DE MEDICINA DE PATENte y medicina tradicional, Coatepec,Veracruz, 1994

prácticas de las madres entrevistadas o el hecho de tener el concepto de deshidratación, por el otro.

\section{Significados de las acciones}

El significado más común, y que aparece como una representación social muy estructurada que se repite constantemente, es el de la deshidratación como algo "mágico": "es peligrosa", "mata", pero no se entiende qué es. Otras representaciones sociales aparecen como visiones acerca de la enfermedad, sobre todo de lo que causa la diarrea. Por ejemplo, "susto", "mal de 
ojo", "algo que comió y se le pegó". Estas visiones no parecen tener un patrón tan definido como las prácticas curativas que sí aparecen con claridad reproduciéndose en las redes sociales mediante la actitud de compartir "recetas" para curar. Así, la práctica de uso del SHO no obedece, en general, a un significado claro por parte de la madre sobre su función de prevenir la deshidratación. Dentro del grupo de madres que sí usaron el SHO, 64\% expresó que lo hacía porque era bueno para el niño pues contribuía a que éste mejorara y sanara; $3 \%$ dijo que lo había dado pero que no notaba nada y/o que sentía que no servía. Un tercer grupo (33\%) dijo que se lo daba al niño aunque supiera feo porque le hacía bien. Se observó aquí que aun cuando las madres tenían el concepto correcto de deshidratación, desconocían que la función del suero es establecer la reabsorción de líquido en el intestino.

El grupo de madres que no utilizó el SHO también expresó significados que explicaban la acción de no proporcionarlo. Estos significados también tendieron a repetirse, aunque sin un patrón definido. Del total de 144 madres, 19\% dijo no haberlo dado porque no lo tenía; $14 \%$ dijo preferir otros líquidos, en especial la "coca-cola", el "tehuacán" o los "tés". El grupo más grande se ubicó en la razón del "mal sabor" para no haber dado el SHO al niño (49\%). Hubo una madre que no se lo administró porque "antes se lo había dado y lo vomitaba". Hubo un grupo de cinco madres que dijo no conocerlo, y 17 madres (12\%) afirmaron que no lo creían necesario, que no le tenían fe o que no contaban con el dinero para comprarlo. Estas madres no tenían el concepto de deshidratación ni tampoco sabían cuál es la función del suero.

El grupo de madres que no atribuye el significado de "pérdida de líquidos" a la deshidratación, considera que ésta es equivalente a los síntomas de la diarrea y no a un proceso derivado de ella -que tiene sus propios síntomas y que hay que atender por separado, aumentando líquidos y garantizando su absorción mediante el SHO.

Los significados expresados para recurrir a la medicina tradicional están relacionados con tradiciones familiares, o bien, se refieren a actos de fe o a que este tipo de medicina se considera como un recurso inexistente en las instituciones de salud: "así curan las abuelitas", "le tengo fe" o "vamos a curar empacho y caída de mollera que los doctores no saben cómo curar". Sin embargo, el significado que más destacó fue el de "es más barato".

Se observó que a la par que disminuye el uso de la medicina tradicional y aumenta el de la automedica- ción, el uso de la primera tiene un significado de bajo costo. Este significado podría tenerlo también el SHO (es gratuito) y llenar el vacío que deja la alternativa de usar medicamentos costosos y contraindicados; lo mismo podría pasar con los tés como opción barata.

Los significados que destacaron las madres en relación con la práctica de medicación incorrecta son: "cura la diarrea", "es muy efectivo", "ya se lo han recetado", "se ha curado otras veces" y "a otros niños los ha curado". Se trató de significados que hacen referencia a la capacidad que tiene la medicina de curar y a la experiencia de que ha servido en otros casos.

Las madres utilizan diferentes medicinas de acuerdo con experiencias previas o recomendaciones de familiares o vecinos. A esos medicamentos se les confiere un "poder curativo" proveniente de una relación imaginaria entre el momento en que cesan las evacuaciones -casi siempre entre tres y cinco días después de que éstas inician- y el remedio que se proporcionó antes de que esto sucediera.

Las madres no conciben la diarrea aguda como un episodio autolimitado; en cambio, tenían mucha claridad respecto a la "gripa", que en un momento de la entrevista se introducía con un afán comparativo. El hecho de haber experimentado como curativa la relación entre medicamento-fin de evacuaciones condiciona que en próximos episodios se privilegie esa medicina específica. Cuando esto no resulta (ya que no necesariamente vuelve a coincidir la toma de la medicina con la terminación de las evacuaciones), se presenta el patrón de utilización de todo lo que esté a la mano, siempre con un elemento importante: desconocimiento de lo que está sucediendo y de lo que tendría que suceder como resultado (evitar la deshidratación).

Este significado podría revertirse explicando que la medicina no "cura", sino que la enfermedad se cura sola y que la administración de un medicamento puede coincidir con el hecho de que se quite la diarrea, sin que esto signifique que haya una relación causal.

Se observó que los significados que las madres atribuyen a sus prácticas mostraron patrones comunes que tienden a cambiar de acuerdo con las distintas cuadras de la ciudad seleccionadas en la muestra. Existe una especie de "patrón de uso por zonas", especialmente en el tipo de antibiótico preferido. Las madres que no utilizaron el SHO se precian de tener una buena medicina para que la diarrea se "corte". Asimismo, se precian de recomendarlo a las vecinas y parientes con el respaldo de su experiencia. Es como si poseer estos saberes les diera una especie de "poder" ante los demás, ya que sienten que su conocimiento está ava- 
lado por la experiencia. En efecto, este saber es una representación de la realidad basada en las experiencias que han tenido, ya sea con sus hijos o con los hijos de parientes o amigas; el problema es que la construcción del entramado de significados se da con base en una experiencia empírica interpretada a partir de un marco explicativo erróneo: que la práctica curativa corta y cura la diarrea -ya que muchas veces efectivamente coinciden el hecho de parar la diarrea (se autolimita) y el de haber usado un medicamento.

El entramado de significados se reproduce como representación social y se va convirtiendo en un patrón compartido por medio de las redes sociales de apoyo. Estos significados, junto con las prácticas de escaso uso del SHO y del uso de medicina, son obstáculos para la administración del suero porque aparecen como los recursos necesarios para curar la diarrea. $\mathrm{Al}$ no tener el significado de que la deshidratación es un proceso de pérdida de líquidos, no se ejerce la acción de aumentar la administración de los mismos; y al desconocer la función del SHO de restablecer la absorción, éste no aparece como la terapia adecuada para prevenir el proceso que pone en peligro al niño. Lo angustiante para las madres es que el niño siga teniendo diarrea, y entonces las acciones tienden a "pararla" o "taparla". Ante la imperante urgencia de parar la diarrea, se privilegian precisamente las prácticas que hay que desalentar.

Se puede concluir que el hecho de que muchas madres no conciban la deshidratación como pérdida de líquidos y de que ninguna sepa que la función del suero es permitir la absorción hasta que la enfermedad se cure sola, está estrechamente ligado a las prácticas de no utilizar el SHO regularmente y recurrir a medicamentos contraindicados. Esta situación de falta de información y de carencia de un marco de significados adecuados que obstaculiza la utilización de la terapia de rehidratación oral podría enfrentarse con programas educativos dirigidos específicamente a dar significados claros que redunden en experiencias positivas y que posteriormente se reproduzcan en el tejido social.

Los significados reflexionados por las madres en relación con sus acciones tendieron a ser bastante homogéneos y se repitieron con mucha regularidad, lo cual permitió la codificación, el proceso de categorización y la interpretación. Se observó que los significados mostraron la doble característica de la intersubjetividad: son individuales y al mismo tiempo pertenecen a condiciones sociales y culturales específicas. Son producto de la experiencia pero abrevan de representa- ciones sociales comunes que se reproducen por medio de las prácticas.

\section{Discusión}

En este trabajo se encontraron porcentajes de uso del SHO y de medicamentos contraindicados similares a los de la encuesta nacional de $1993 .{ }^{4}$ Asimismo, se confirmó que las acciones de las madres no obedecen a un entramado de significaciones adecuado para atender el episodio diarreico (como se había descrito en las investigaciones cualitativas) $)^{5,9}$ y que este patrón está generalizado en la población de Coatepec. Además de que la relación entre tener el concepto correcto de deshidratación y usar el SHO es positiva, también se observó que ninguna de las entrevistadas conocía la función del SHO en cuanto a que propicia la absorción intestinal de líquidos.

Debido a que las prácticas terapéuticas y los significados parecían ser similares en las distintas cuadras donde se efectuaron las entrevistas, independientemente del tipo de colonia o de vivienda, es importante hacer un estudio de cómo se reproducen estos patrones de significancia por medio de las redes de ayuda, más que profundizar en relaciones causales entre variables socioeconómicas y prácticas de la población. ${ }^{7,8}$

Fue imposible establecer una separación clara entre automedicación en la familia y medicación incorrecta prescrita por proveedores de servicios de salud, ya que muchas veces la medicina recetada para episodios previos se vuelve a usar o a comprar. Por otra parte es difícil obtener este tipo de datos, pues las madres saben que no deberían automedicar a los niños. Otras veces el medicamento se pide en la farmacia por recomendación de alguien (quien a su vez lo usó por prescripción médica en algún momento). Es importante estudiar a fondo la relación de las prácticas en el hogar con las de los médicos, farmacéuticos e instituciones de salud (sobre todo la práctica de acudir al médico pero solamente a comprar la medicina, sin que éste vea al niño) porque, al parecer, éstos están coadyuvando a reproducir las prácticas contraindicadas de manera importante. El estudio acerca de cómo participan los profesionales de la salud en la reproducción de significados dentro de las redes sociales puede servir para que la capacitación que actualmente les proporciona el sector salud (contemplada en el Programa Nacional de Control de Diarreas) se complemente con la educación a la población y se materialice en prácticas que se reproduzcan socialmente y que, en efecto, prevengan la deshidratación en los niños. 


\section{Referencias}

1. Servicios Coordinados de Salud Pública en el Estado de Veracruz. Programa Estatal de Atención Materno Infantil. Xalapa: Secretaría de Salud Pública en el Estado de Veracruz, 1993.

2. Instituto $N$ acional de Estadística, Geografía e Informática. Estadísticas vitales de 1993 por estados. México, D.F.: D irección General de Estadística, IN EGI, 1994.

3. Servicios Coordinados de Salud Pública en el Estado de Veracruz. Programa Estatal de Control de Enfermedades Diarreicas. X alapa: Secretaría de Salud Pública en el Estado de Veracruz, 1994:9.

4. Dirección General de Epidemiología. Encuesta sobre el manejo efectivo de los casos de diarrea en el hogar. México, D.F.: Consejo $\mathrm{N}$ acional del Control de Enfermedades Diarreicas, Secretaría de Salud, 1994.

5. A ceituno A, Alvarez $S, G$ arcía V, Juárez $S$, Luna T, Méndez N A, et al. Manejo en el hogar de situaciones de diarrea infantil complicada y redes sociales de apoyo, 1994. Dimensión Antropológica 1995;5:83-104.

6. O rganización Mundial de la Salud. El uso racional de medicamentos contra la diarrea en la infancia. Foro Mundial de la Salud 1992;12(3): 382383.
7. Uribe F, Hernández $\mathrm{R}, \mathrm{N}$ avarro A, Tello A, Benítez 0, Cravioto A. Patrones de aceptación de la terapia de hidratación oral a nivel domiciliario en una cohorte de madres rurales. Bol Med Hosp Infant Mex 1991;48(5): 320-325.

8. Leal M, Silva R, Gama S. Percepçäo materna da crianças com diarréia. Estudo de concordância com diagnòstico médico. Rev Saude Publica 1990;24(3):196-203.

9. Berman P, Kendall K, Bhattacharyya K. The household production of health: Integrating social science perspectives on micro-level health determinants. Soc Sci Med 1994;38(2):205-215.

10. Alvarez $S$, Correa E, Morales ME. Las madres ante la diarrea y la deshidratación infantil. México, D.F: Instituto $\mathrm{N}$ acional de Antropología e Historia, Colección Científica,1995.

11. Schütz, A. La construcción significativa del mundo social. Barcelona: Paidós, 1993.

12. G rinberg, M. Problemas conceptuales en antropología médica. ¿N uevos paradigmas? En: C iencias Sociales y Medicina. La Salud en Latino américa. Buenos Aires: Universidad de Buenos Aires, 1992:134. 